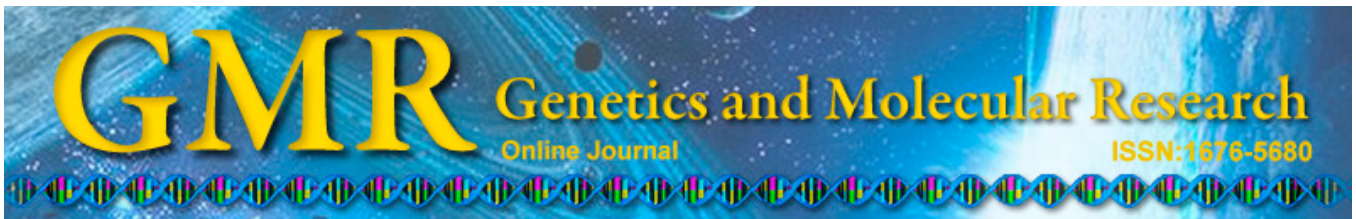

\title{
Expression of genes coding for selected amino acid transporters in small intestine, liver, and skeletal muscle of pigs fed excess branched-chain amino acids
}

M. Cervantes ${ }^{1}$, N. Arce ${ }^{1}$, H. García ${ }^{1}$, M. Cota $^{1}$, J.K. Htoo ${ }^{2}$ and A. Morales ${ }^{1}$

${ }^{1}$ Instituto de Ciencias Agrícolas, Universidad Autónoma de Baja California, Mexicali, México

${ }^{2}$ Evonik Industries AG, Nutrition Research, Hanau, Germany

Corresponding author: A. Morales

E-mail: adriana_morales@uabc.edu.mx

Genet. Mol. Res. 14 (3): 9779-9792 (2015)

Received January 31, 2015

Accepted May 26, 2015

Published August 19, 2015

DOI http://dx.doi.org/10.4238/2015.August.19.11

\begin{abstract}
Excess Leu reduces the availability of Ile and Val in pigs likely by reducing absorption of the latter amino acids (AA). Twenty-four crossbred pigs were used to evaluate the effect of excess Leu alone or with surplus Ile and Val on the expression of cationic $\left(\mathrm{b}^{0},{ }^{+} \mathrm{AT}\right.$ and CAT1) and neutral $\left(\mathrm{B}^{0} \mathrm{AT} 1\right) \mathrm{AA}$ transporters in the small intestine, liver, and skeletal muscle. Dietary treatments included: 1) basal diet; 2) basal plus $0.43 \%$ L-Leu (excess Leu); 3) basal plus $0.43 \%$ Leu, $0.20 \%$ L-Ile, and $0.25 \%$ L-Val (excess Leu, Ile, and Val). The basal diet contained $0.90 \%$ standardized ileal digestible Lys, as well as crystalline L-Lys, L-Thr, DL-Met, L-Trp, L-Leu, L-Ile, L-His, and L-Val. Diets 2 and 3 contained 52\% more Leu and diet 3 contained $42 \%$ more Ile and Val compared with the basal diet. Excess Leu or excess Leu, Ile, and Val reduced $\mathrm{b}^{0},{ }^{+} \mathrm{AT}$ expression in the jejunum (P $<0.05)$ but had no effect in the ileum and liver. Excess Leu increased CAT1 expression in the ileum but reduced expression in the liver $(\mathrm{P}<$
\end{abstract}


0.05). Excess Leu, Ile, and Val increased $(\mathrm{P}<0.05) \mathrm{B}^{0} \mathrm{AT} 1$ expression in the jejunum and tended to increase $(\mathrm{P}=0.10)$ expression in the ileum. In general, $\mathrm{b}^{0},{ }^{+} \mathrm{AT}$ expression was higher but CAT1 expression was lower in the jejunum than in the ileum; $\mathrm{B}^{0} \mathrm{AT} 1$ was similarly expressed in the jejunum and ileum. Excess Leu or any branchedchain AA affects AA transporter expression, which may affect the absorption and availability of AA for animal growth.

Key words: Amino acid transporter; Branched-chain amino acid; Pigs; Gene expression

\section{INTRODUCTION}

Practical diets for pigs contain excess levels of some amino acids (AA) such as Leu, which is well documented to interact with the other 2 branched-chain AA (BCAA), Ile and Val. Large excess dietary Leu markedly reduced the serum concentration (SC) of Ile and Val in weanling pigs (Edmonds and Baker, 1987; Gatnau et al., 1995). Moderate excess dietary Leu $(150 \%)$ also reduced the SC of Ile and Val in growing pigs (Langer et al., 2000). Decreased SC of Ile and Val because of excess dietary Leu have been attributed to increased activities of branched-chain keto acid dehydrogenase enzymes (Langer et al., 2000; Wiltafsky et al., 2010). All 3 BCAA share this enzyme complex in their degradative pathways (Block, 1989), and excess Leu increases the catabolism of all BCAA (Block and Harper, 1984). Similarly, BCAA share common transport systems for absorption in the small intestine (Bröer, 2008), suggesting that Ile and Val compete with Leu for absorption. Hence, differences in SC of BCAA because of excess dietary Leu may also result from competition for intestinal absorption.

$\mathrm{B}^{0} \mathrm{AT} 1$ is the major transporter for BCAA in the small intestine (Bröer et al., 2004). It is confined to the apical membrane of enterocytes with equal preference for all 3 BCAA (Bröer, 2008), suggesting that an excess of 1 BCAA may impair the absorption of the other 2. Thus, excess Leu appears to not only increases the cellular catabolism of Ile and Val, but also reduces their intestinal absorption. The interaction between dietary Leu and Lys for intestinal absorption has been documented. Transport of Lys across the apical membrane of enterocytes is primarily facilitated by the AA transporter $\mathrm{b}^{0},{ }^{+} \mathrm{AT}$ (Bröer et al., 2004) and with lower activity by the CAT1 transport system. The transporter $\mathrm{b}^{0}{ }^{+} \mathrm{AT}$ exchanges Lys for neutral AA, particularly Leu (Bröer et al., 2004; Majumder et al., 2009), leading to intestinal absorption of Lys by $b^{0}{ }^{+}$AT that is coupled with the efflux of Leu (Pineda et al., 2004). We recently observed reduced expression of $\mathrm{b}^{0}{ }^{+} \mathrm{AT}$ in growing pigs (García-Villalobos et al., 2012; Morales et al., 2013) fed excess Leu diets, and surplus Ile failed to correct the reduction (Cervantes-Ramírez et al., 2013). However, there is limited information regarding the effect of adding surplus Ile and Val to diets with excess Leu on the abundance of transport systems for BCAA and cationic AA (Lys) in growing pigs.

We hypothesized that excess dietary Leu affects the abundance of AA transporters, which may partially explain the lower SC of Ile, Val, and Lys in pigs (Cervantes-Ramírez et al., 2013). We also hypothesized that surplus Ile and Val may correct the effect of excess dietary Leu. Although AA absorption is expected to take place in the jejunum (Silk et al., 1985), it is unknown whether excess Leu or all BCAAs in the diet modifies the site of AA absorption. In this study, we evaluated the effect of excess Leu alone or in combination with surplus Ile 
and Val on the expression of the AA transport systems $\mathrm{b}^{0},{ }^{+} \mathrm{AT}, \mathrm{CAT} 1$, and $\mathrm{B}^{0} \mathrm{AT} 1$ in the small intestine, liver, and skeletal muscle of growing pigs. In addition, AA transporter expression in the jejunum was compared to that in the ileum. We demonstrate the varied effect of excess dietary Leu alone or with surplus Ile and Val on the expression of AA transporters in different tissues.

\section{MATERIAL AND METHODS}

The pigs used in this experiment were cared for in accordance with the guidelines established in Official Mexican Regulations on Animal Care (Norma Oficial Mexicana, 2001). A 21-day growth trial was conducted with 24 crossbred pigs (Large White x Duroc) with initial body weights of $31.8 \pm 1.2 \mathrm{~kg}$, which were randomly assigned to 3 dietary treatments. There were 8 pig replicates ( 2 barrows and 6 gilts). The initial body weight was balanced across pens. Pigs were individually housed in raised floor metabolism pens (1.2 x $1.2 \times 1.0 \mathrm{~m}$ ) equipped with a nipple water drinker and iron mesh floor in a temperaturecontrolled room $\left(22^{\circ}-24^{\circ} \mathrm{C}\right)$. All pigs were trained to consume similar amounts of feed each day, which was achieved by restricting their intake to the amount consumed by the pig with the lowest feed intake, which was adjusted every week. All pigs were fed twice, at 7:00 and 19:00 $\mathrm{h}$, during the entire trial.

\section{Dietary treatments}

A basal diet was formulated with wheat as the sole major ingredient, the most common ingredient used under practical conditions in northern México, and supplemented with crystalline AA (Table 1). Dietary treatments were: 1) basal diet; 2) basal diet plus $0.43 \%$ L-Leu (excess Leu); 3) same as in 2 but added with $0.20 \%$ L-Ile and $0.25 \%$ L-Val [excess Leu, Ile and Val (LIV)]. The basal diet was formulated to contain $0.90 \%$ standardized ileal digestible Lys using the analyzed AA content (Table 2) and standardized ileal digestible coefficients in wheat (Stein et al., 2001). Crystalline L-Lys, L-Thr, DL-Met, L-Trp, L-Leu, L-Ile, L-His, and L-Val were added to the basal diet to create essential AA:Lys ratios close to the ideal protein level for growing pigs (NRC, 2012). The addition of L-Leu to diets 2 and 3 increased the Leu content by $52 \%$ compared to the basal diet. Additionally, diet 3 contained approximately $42 \%$ more Ile and Val compared to the basal diet. All diets were supplemented with a mineral-vitamin premix to meet or exceed their requirements for growing pigs (NRC, 2012) and contained 10.4 megajoules of net energy $/ \mathrm{kg}$.

\section{Tissue collection}

At the end of the experiment, all pigs were euthanized by electrical stunning and exsanguination for tissue collection $2.5 \mathrm{~h}$ after their last feeding at 7:00 h. The corpses were immediately eviscerated and mucosal samples scratched from the middle jejunum and ileum; approximately $0.5 \mathrm{~g}$ was collected into $2-\mathrm{mL}$ microtubes. Samples from the liver and the longissimus dorsi (LD) muscle (approximately 0.5-1.0 g) were also collected. All samples were immediately frozen in liquid nitrogen and stored at $-82^{\circ} \mathrm{C}$ until analysis. The total collection process took no longer than $8 \mathrm{~min}$ to maximize the quality of the extracted RNA. 
Table 1. Composition of experimental diets (as-fed).

\begin{tabular}{|c|c|c|c|}
\hline & \multicolumn{3}{|c|}{ Dietary treatment } \\
\hline & Basal & Excess Leu & Excess Leu + Ile + Val \\
\hline \multicolumn{4}{|l|}{ Ingredient (\%) } \\
\hline Wheat & 95.08 & 95.08 & 95.08 \\
\hline Corn starch & 0.88 & 0.45 & - \\
\hline L-Lys.HCl & 0.79 & 0.79 & 0.79 \\
\hline L-Thr & 0.27 & 0.27 & 0.27 \\
\hline DL-Met & 0.13 & 0.13 & 0.13 \\
\hline L-Trp & 0.08 & 0.08 & 0.08 \\
\hline L-Leu & 0.10 & 0.53 & 0.53 \\
\hline L-Ile & 0.13 & 0.13 & 0.33 \\
\hline L-His & 0.03 & 0.03 & 0.03 \\
\hline L-Val & 0.16 & 0.16 & 0.41 \\
\hline Calcium carbonate & 1.40 & 1.40 & 1.40 \\
\hline Dicalcium phosphate & 0.40 & 0.40 & 0.40 \\
\hline Iodized salt & 0.35 & 0.35 & 0.35 \\
\hline Vitamin and Mineral Premix & 0.20 & 0.20 & 0.20 \\
\hline \multicolumn{4}{|c|}{ Calculated SID content of amino acids (\%) } \\
\hline Arg & 0.51 & 0.51 & 0.51 \\
\hline His & 0.29 & 0.29 & 0.29 \\
\hline Ile & 0.50 & 0.50 & 0.70 \\
\hline Leu & 0.83 & 1.26 & 1.26 \\
\hline Lys & 0.90 & 0.90 & 0.90 \\
\hline Met & 0.30 & 0.30 & 0.30 \\
\hline Phe & 0.50 & 0.50 & 0.50 \\
\hline Thr & 0.57 & 0.57 & 0.57 \\
\hline $\operatorname{Trp}$ & 0.21 & 0.21 & 0.21 \\
\hline Val & 0.62 & 0.62 & 0.87 \\
\hline
\end{tabular}

Table 2. Analyzed composition of experimental diets (as-fed).

\begin{tabular}{lccc}
\hline Amino acid (\%) & \multicolumn{3}{c}{ Dietary treatment } \\
\cline { 2 - 4 } & Basal & Excess Leu & Excess Leu + Ile + Val \\
\hline Indispensable & & & 0.57 \\
Arg & 0.57 & 0.57 & 0.30 \\
His & 0.29 & 0.30 & 0.70 \\
Ile & 0.51 & 0.52 & 1.25 \\
Leu & 0.87 & 1.23 & 0.94 \\
Lys & 0.88 & 0.90 & 0.30 \\
Met & 0.30 & 0.29 & 0.54 \\
Phe & 0.53 & 0.54 & 0.57 \\
Thr & 0.56 & 0.58 & 0.21 \\
Trp & 0.22 & 0.22 & 0.89 \\
Val & 0.64 & 0.66 & \\
Dispensable & & & 0.41 \\
Ala & 0.41 & 0.42 & 0.62 \\
Asp & 0.62 & 0.62 & 0.24 \\
Cys & 0.25 & 0.25 & 3.08 \\
Glu & 3.02 & 3.09 & 0.43 \\
Gly & 0.43 & 0.43 & 1.11 \\
Pro & 1.09 & 1.1 & 0.53 \\
Ser & 0.53 & 0.53 & \\
\hline
\end{tabular}

\section{RNA extraction and purification}

The samples of intestinal (jejunum and ileum) mucosa, liver, and LD were treated to extract total RNA using Trizol reagent (Invitrogen, Carlsbad, CA, USA) as reported by Méndez et al. (2011). Purified RNA was then eluted with $30 \mu \mathrm{L}$ RNase-free distilled water and 
stored at $-82^{\circ} \mathrm{C}$. The concentration of total RNA was determined spectrophotometrically at 260 nm (Helios $\beta$, Thermo Electron Co., Waltham, MA, USA), and RNA purity was assessed based on the $\mathrm{A}_{260} / \mathrm{A}_{280}$ ratio, which ranged from 1.8-2.0 (Sambrook and Russell, 2001). The integrity of total RNA was evaluated by gel electrophoresis on $1 \%$ agarose gels. All RNA samples showed good quality, with a 28S:18S rRNA ratio of approximately 2.0:1 (Sambrook and Russell, 2001).

\section{Reverse transcription}

Approximately $2 \mu \mathrm{g}$ total RNA was treated with $1 \mathrm{U} / \mu \mathrm{L}$ DNase I (Invitrogen) and 6 $\mu \mathrm{L} 5 \mathrm{X}$ reverse transcription buffer in a $30-\mu \mathrm{L}$ reaction conducted in diethylpyrocarbonatetreated water; the reaction was carried out for $15 \mathrm{~min}$ at room temperature and then placed for 15 min at $70^{\circ} \mathrm{C}$ to stop the reaction. Reverse transcription was initiated with DNase-treated RNA samples, adding $1 \mu \mathrm{L} 150 \mu \mathrm{g} / \mathrm{mL}$ random primers (Invitrogen) and $1 \mu \mathrm{L} 10 \mu \mathrm{M}$ dNTPs. The reaction was incubated at room temperature and then placed on ice for $1 \mathrm{~min} ; 3 \mu \mathrm{L} 0.1$ $\mathrm{M}$ dithiothreitol, $1 \mu \mathrm{L} 40 \mathrm{U} / \mu \mathrm{L}$ ribonuclease inhibitor (RNaseOUT, Invitrogen), and $2 \mu \mathrm{L} 5 \mathrm{X}$ reverse transcription buffer were added to the reaction, which was incubated at $42^{\circ} \mathrm{C}$ for 2 min to stabilize the reaction before adding $1 \mu \mathrm{L} 200 \mathrm{U} / \mu \mathrm{L}$ reverse transcriptase enzyme (RTSuperscript III, Invitrogen). The reaction was incubated at $42^{\circ} \mathrm{C}$ for $50 \mathrm{~min}$. The mixture was incubated at $70^{\circ} \mathrm{C}$ for $15 \mathrm{~min}$ and then placed on ice to stop the reaction. cDNA samples were quantified spectrophotometrically and diluted into a final concentration of $50 \mathrm{ng} / \mu \mathrm{L}$.

\section{Quantitative polymerase chain reaction (qPCR)}

Specific primers for each AA transporter mRNA were designed according to their published sequences in GenBank (Table 3). Additionally, a housekeeping 18S rRNA gene was used as an endogenous control to normalize variations in mRNA because its expression is very stable (Liao et al., 2009). Before conducting the reaction, end-point PCR was carried out to standardize the amplification conditions for each pair of primers. In order to confirm the specificity of the PCR products for its mRNA, a sample of each PCR product was purified using the PureLink PCR Purification kit (Invitrogen) and sequenced at the Davis Sequencing Facility (Davis, CA, USA). The sequencing results revealed that the products for $\mathrm{b}^{0},{ }^{+} \mathrm{AT}, \mathrm{CAT} 1$, $\mathrm{B}^{0} \mathrm{AT} 1$, and $18 \mathrm{~S}$ rRNA showed $100 \%$ homology with their corresponding expected sequences acquired from the virtual template sequences reported in GenBank.

Expression of AA transporters in the jejunum, ileum, liver, and LD was estimated by qPCR assays, using the Maxima SYBR Green/ROX qPCR Master Mix (Fermentas, Vilnius, Lithuania) into a Chromo 4-DNA Engine with the MJ Opticon Monitor 3.1 software (Bio-Rad, Hercules, CA, USA). The equipment was calibrated by constructing a standard curve using the $18 \mathrm{~S}$ rRNA cloned into a TOPO vector 4.0, from which a calibrator cDNA was produced. The standard curve was obtained using known concentrations of 100 -fold serial dilutions of the cDNA. qPCR contained $50 \mathrm{ng}$ cDNA, $0.5 \mu \mathrm{M}$ of each specific primer, $12.5 \mu \mathrm{L} 2 \mathrm{X}$ SYBR green/ ROX qPCR Master Mix, and DNase/RNase-free water for a final volume of $25 \mu \mathrm{L}$. 18S rRNA was used as housekeeping gene to standardized the amount of amplified DNA. The PCR conditions used for the amplification and quantification were an initial denaturation stage at $95^{\circ} \mathrm{C}$ for 1 min, followed by 45 cycles of amplification with denaturation at $95^{\circ} \mathrm{C}$ for $30 \mathrm{~s}$, annealing at $56^{\circ} \mathrm{C}$ for $15 \mathrm{~s}$, and extension at $72^{\circ} \mathrm{C}$ for $30 \mathrm{~s}$, followed by a melting curve program $\left(60^{\circ}\right.$ to $\left.90^{\circ} \mathrm{C}\right)$. Fluorescence was measured at the end of every cycle and every $0.2^{\circ} \mathrm{C}$ during the melting program. 
Table 3. Primers for qPCR analyses of cDNA derived from mRNA of amino acid transporters $b^{0},{ }^{+} A T, C A T 1$, $\mathrm{B}^{0} \mathrm{AT} 1$, and $18 \mathrm{~S}$ ribosomal RNA (18S rRNA).

\begin{tabular}{|c|c|c|c|c|}
\hline mRNA & Primer & Location (bp) on the template & Sequence & Amplicon size (bp) \\
\hline \multicolumn{5}{|c|}{$\mathrm{b}^{0+} \mathrm{AT}$ : Sus scrofa cationic amino acid transporter SLC7A9, mRNA (GenBank: EF127857) } \\
\hline & Forward & $1-19$ & 5'-CGGAGAGAGGATGAGAAGT-3' & \multirow[t]{2}{*}{562} \\
\hline & Reverse & $545-562$ & 5'-GCCCGCTGATGATGATGATGA-3' & \\
\hline \multicolumn{5}{|c|}{ CAT1: Sus scrofa cationic amino acid transporter-1 mRNA (GenBank: AY371320) } \\
\hline & Forward & $4239-4258$ & 5'-GTCGGTTGCAAAGACCATTT-3' & \multirow[t]{2}{*}{329} \\
\hline & Reverse & $4548-4567$ & 5'-GAGCGGTGCTGACAACAGTA-3' & \\
\hline \multicolumn{5}{|c|}{$\mathrm{B}^{0} \mathrm{AT} 1$ : Sus scrofa neutral amino acid transporter SLC6A19 mRNA (GenBank: DQ231579.1) } \\
\hline & Forward & $8-28$ & 5'-TCTGTCCACAACAACTGCGAG-3' & \multirow{2}{*}{205} \\
\hline & Reverse & $193-212$ & 5'-CAGCGAAGTTCTCCTGCGTC-3' & \\
\hline \multicolumn{5}{|c|}{ 18S rRNA: Sus scrofa 18 S ribosomal RNA gene (GenBank: AY265350) } \\
\hline & Forward & $236-255$ & 5'-GGCCTCACTAAACCATCCAA-3' & \multirow[t]{2}{*}{295} \\
\hline & Reverse & $511-530$ & 5'-TAGAGGGACAAGTGGCGTTC-3' & \\
\hline
\end{tabular}

\section{Statistical analysis}

Analyses of variance of the data were performed using the GLM of SAS (Statistical Analysis System 9.1, SAS Institute, Cary, NC, USA). Two contrasts (C) were constructed to test the effect of excess Leu alone or in combination with Ile plus Val in the basal diet: $\mathrm{C}_{1}$, basal vs excess Leu; $\mathrm{C}_{2}$, basal vs excess LIV. Probability levels of $\mathrm{P} \leq 0.05$ and $0.05<\mathrm{P} \leq 0.10$ were defined as significant differences and tendencies, respectively.

\section{RESULTS}

All pigs remained healthy during the experiment and consumed their daily rations within $30 \mathrm{~min}$. Average daily feed intake per pig during weeks 1, 2, and 3 was $1.60,1.65$, and $1.76 \mathrm{~kg}$, respectively; the average feed intake during the whole study was $1.70 \mathrm{~kg} / \mathrm{day}$.

\section{Expression of $\mathrm{b}^{0},{ }^{+} \mathrm{AT}, \mathrm{CAT} 1$, and $\mathrm{B}^{0} \mathrm{AT} 1$ in the jejunum and ileum}

The expression levels of $\mathrm{b}^{0}, \mathrm{AT}, \mathrm{CAT} 1$, and $\mathrm{B}^{0} \mathrm{AT} 1$ in the jejunum and ileum of pigs fed the basal diet or the basal with excess Leu or with excess LIV are presented in Figure 1. In the jejunum, expression of $\mathrm{b}^{0},{ }^{+} \mathrm{AT}$ was lower in pigs fed excess Leu $(\mathrm{P}=0.013)$ or excess LIV $(\mathrm{P}<0.001)$ diets than in pigs fed the basal diet. In the ileum, there was no effect of excess Leu $(\mathrm{P}=0.161)$ or excess LIV $(\mathrm{P}=0.216)$ on $\mathrm{b}^{0}{ }^{+} \mathrm{AT}$ expression. The expression of CAT1 in the jejunum was not affected by Leu or excess LIV $(\mathrm{P}>0.10)$. In contrast, CAT1 expression in the ileum was higher in pigs fed the excess Leu diet $(\mathrm{P}=0.017)$, but no difference was observed between pigs fed the basal and the excess LIV diet $(\mathrm{P}=0.850) . \mathrm{B}^{0} \mathrm{AT} 1$ expression was not affected in the jejunum $(\mathrm{P}=0.313)$ or ileum $(\mathrm{P}=0.384)$ by adding excess Leu to the basal diet; however, excess LIV increased $\mathrm{B}^{0} \mathrm{AT} 1$ expression in the jejunum $(\mathrm{P}=0.011)$ and $\mathrm{B}^{0} \mathrm{AT} 1$ expression tended to increase in the ileum $(\mathrm{P}=0.100)$.

The expression values of $\mathrm{b}^{0}, \mathrm{AT}$, CAT1, and $\mathrm{B}^{0} \mathrm{AT} 1$ in the jejunum as compared to those in the ileum, within each dietary treatment, are presented in Figure 2. Expression of $\mathrm{b}^{0}{ }^{+} \mathrm{AT}$ was higher in the jejunum than in the ileum, regardless of whether pigs were fed the basal or the excess Leu or excess LIV diet $(\mathrm{P} \leq 0.01)$. However, CAT1 expression was markedly higher $(\mathrm{P}<0.01)$ in the ileum than in the jejunum, regardless of the diet. Expression of 
$\mathrm{B}^{0} \mathrm{AT} 1$ did not differ between the intestinal segments of pigs fed either the basal or the excess Leu diet $(\mathrm{P}>0.10)$, but was higher $(\mathrm{P}=0.032)$ in the jejunum of pigs fed the excess LIV diet. Overall, regardless the dietary treatment, the expression of $b^{0}{ }^{+}$AT was markedly higher in the jejunum than in the ileum, but CAT1 expression was higher $(\mathrm{P}<0.01)$ in the ileum. No difference $(\mathrm{P}>0.10)$ was observed in the expression of $\mathrm{B}^{0} \mathrm{AT} 1$ between intestinal segments.

\section{Expression of $\mathrm{b}^{0},{ }^{+} \mathrm{AT}$ and CAT1 in liver and skeletal muscle}

The expression values of $\mathrm{b}^{0},{ }^{+} \mathrm{AT}$ and CAT1 in the liver and the LD muscle of pigs fed the basal, excess Leu, and excess LIV diets are presented in Figure 3. In the liver, $b^{0},{ }^{+}$AT expression was not affected by excess Leu or excess LIV $(\mathrm{P}>0.10)$. CAT1 expression was not affected by excess LIV $(\mathrm{P}=0.302)$; however, expression was reduced by excess Leu $(\mathrm{P}=$ 0.015). Expression of $b^{0},{ }^{+}$AT was not detected in skeletal muscle samples. Expression of CAT1 in the LD muscle was not affected by excess Leu or excess LIV $(\mathrm{P}>0.10)$.
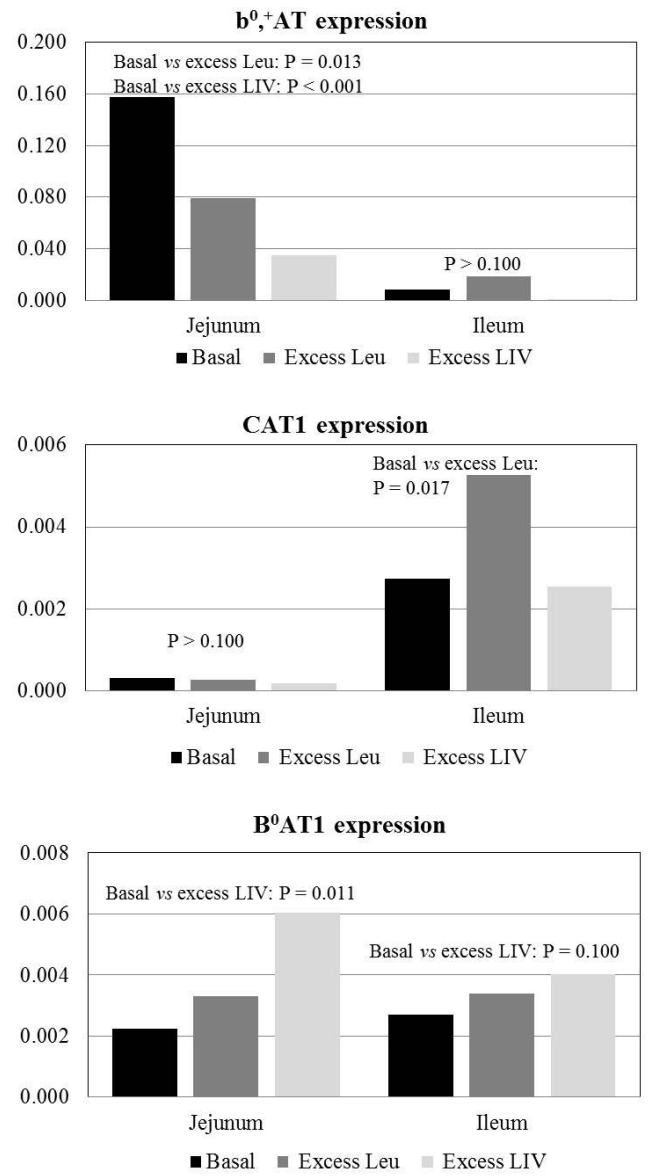

Figure 1. Effect of feeding pigs with a wheat-based diet supplemented with crystalline amino acids (basal), with excess Leu alone (excess Leu), or with excess Leu, Ile, and Val (excess LIV) on expression of amino acid transport systems $\mathrm{b}^{0}{ }^{+} \mathrm{AT}, \mathrm{CAT} 1$, and $\mathrm{B}^{0} \mathrm{AT} 1$ in the jejunum and ileum (arbitrary units, molecule ratio of mRNA:18S $\mathrm{rRNA}$; $\mathrm{N}=8$ ). 

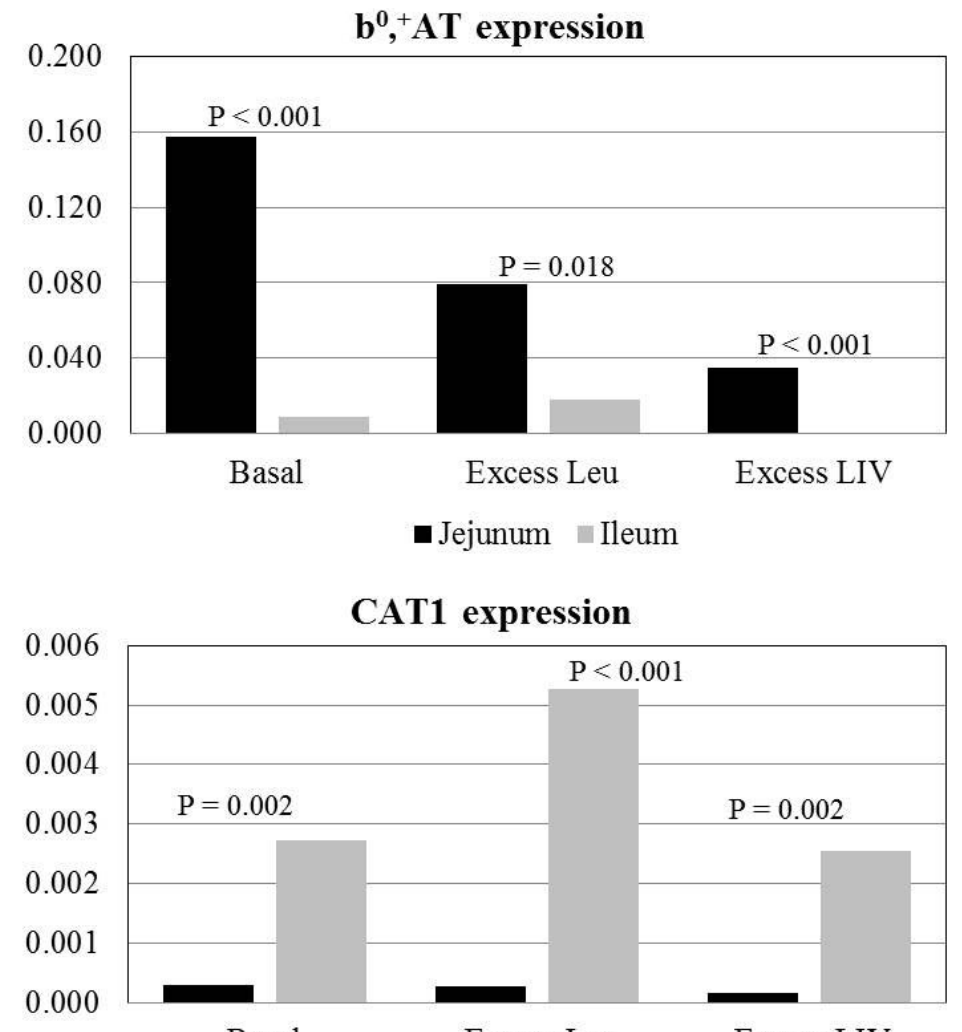

Basal Excess Leu

Excess LIV

- Jejunum Ileum

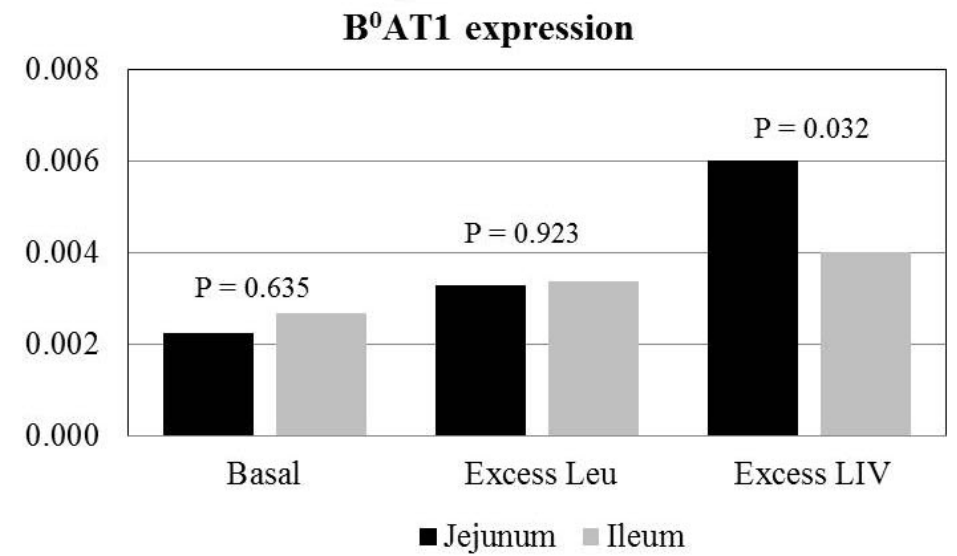

Figure 2. Expression of the amino acid transport systems $b^{0},{ }^{+} \mathrm{AT}, \mathrm{CAT} 1$, and $\mathrm{B}^{0} \mathrm{AT} 1$ in the jejunum compared to the ileum of pigs fed either a wheat-based diet supplemented with crystalline amino acids (basal), with excess Leu alone (excess Leu) or combined with excess Ile and Val (excess LIV; arbitrary units, molecule ratio of mRNA:18S rRNA; $\mathrm{N}=8)$. 


\section{$\mathbf{b}^{0},{ }^{+} \mathbf{A T}$ and CAT1 expression in liver}

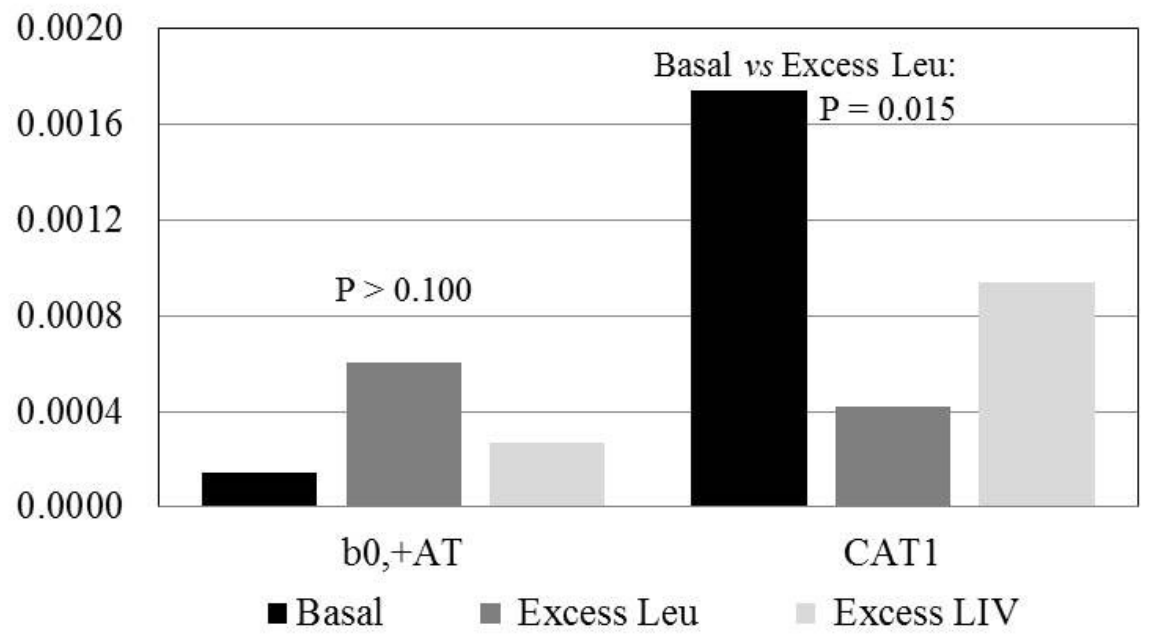

CAT1 expression in longissimus muscle

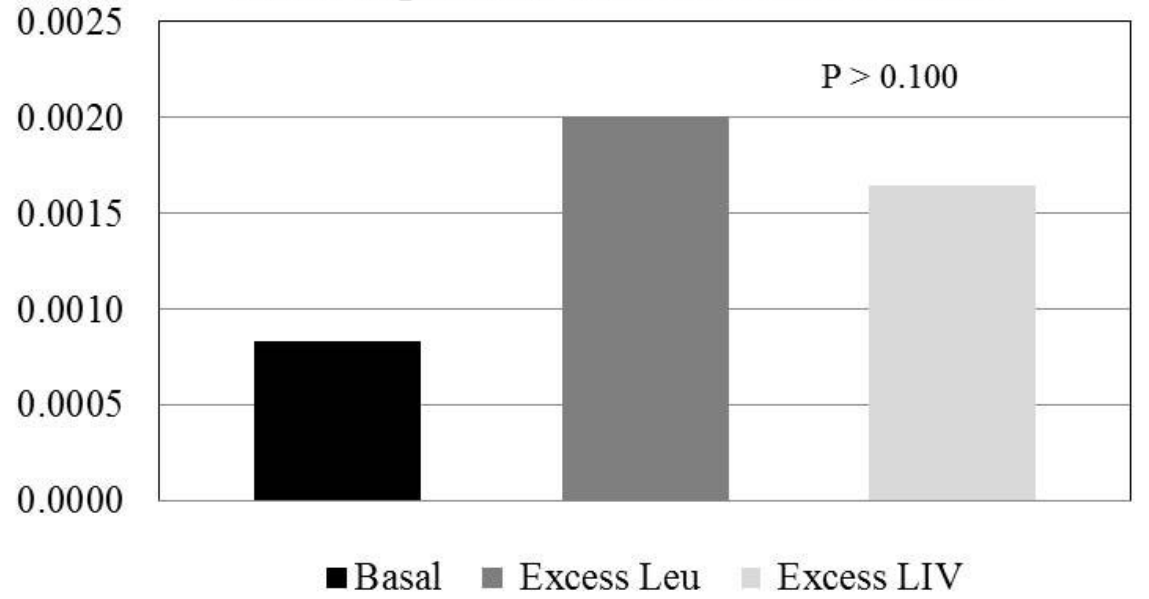

Figure 3. Expression of amino acid transporter systems $b^{0},+A T$ and CAT1 in the liver and the longissimus muscle of pigs fed a wheat-based diet supplemented with crystalline amino acids (basal), with excess Leu alone (excess Leu) or combined with excess Ile and Val (excess LIV; arbitrary units, molecule ratio of mRNA:18S rRNA; $N=8$ ).

\section{DISCUSSION}

The main objective of this study was to determine whether excess Leu alone or combined with surplus Ile plus Val affects the expression of 2 cationic $\left(\mathrm{b}^{0},{ }^{+} \mathrm{AT}\right.$ and CAT1) and 1 neutral $\left(\mathrm{B}^{0} \mathrm{AT} 1\right) \mathrm{AA}$ transporters in the small intestine, liver, and skeletal muscle of growing pigs. To minimize the effect of AA interactions, other than those related to BCAA, a basal diet was formulated containing wheat and crystalline AA to produce an AA pattern close to that in 
ideal protein for pigs (NRC, 2012). The analyzed Ile:Lys, Leu:Lys, and Val:Lys ratios in the basal diet were 58, 98, and 73\%, respectively. The excess Leu diet contained 52\% more Leu than the basal diet, whereas the excess LIV diet contained a $42 \%$ excess of both Ile and Val. Because excess Leu in the diet has been shown to decrease feed intake (Gatnau et al., 1995; Langer et al., 2000; Wiltafsky et al., 2010), all pigs used in this study were trained to have a similar feed intake to avoid any potential effect that differences in AA consumption other than BCAA have on the expression of AA transporters. On average, all pigs consumed $1.70 \mathrm{~kg} /$ day, which was similar to the estimated feed intake of $25-50 \mathrm{~kg}$ pigs (NRC, 2012).

The transport system $\mathrm{B}^{0} \mathrm{AT} 1$ is the major transporter of BCAA in intestine and is confined to the apical membrane of the enterocyte (Bröer et al., 2004), and it is nearly entirely $\mathrm{Na}^{+}$-dependent (Bröer, 2008). According to Bröer (2008), $\mathrm{B}^{0} \mathrm{AT} 1$ transports all neutral AA with different affinities, but Leu, Ile, and Val are equally and the most preferred among all neutral AA, indicating strong competition for absorption between BCAA. A higher content of one BCAA in the intestinal digesta is expected to stimulate its own absorption as compared with other, less concentrated BCAAs. Indeed, several reports (Edmonds and Baker, 1987; Gatnau et al., 1995; Langer et al., 2000) have shown that excess levels of Leu in the diet consistently reduced the SC of Ile and Val in pig. Zhang et al. (2013) reported that $\mathrm{B}^{0} \mathrm{AT} 1$ expression in the jejunum was not affected by feeding weanling pig diets with either deficient or adequate levels of BCAA; however, no studies have demonstrated the effect of excess BCAA on the expression of this transporter. This information is important because practical diets contain excess levels of all 3 BCAA. In the present study, expression of $\mathrm{B}^{0} \mathrm{AT} 1$ in the jejunum and ileum did not differ between pigs fed the excess Leu or the basal diet. These results suggest that higher available Leu content in the excess Leu diet favored Leu absorption, as compared to Ile and Val, which were less concentrated than Leu. This may partially explain the increased SC of Leu and decreased SC of Ile and Val in pigs fed excess Leu diets (Gatnau et al., 1995). Thus, the low SC of Ile and Val reported in pigs fed excess Leu may be attributed not only to increased catabolism of BCAA caused by increased activities of branched-chain keto acid dehydrogenase enzymes (Langer et al., 2000; Wiltafsky et al., 2010), but also to their reduced absorption because of direct competition with high Leu levels. In contrast, excess LIV increased the expression of $\mathrm{B}^{0} \mathrm{AT} 1$ by 2.7 - and 1.5 -fold in the jejunum and ileum, respectively, as compared to those fed the basal diet. This response suggests increased absorption of Ile and Val, which may eventually result in higher $\mathrm{SC}$ of these $2 \mathrm{AA}$, as reported previously (Wiltafsky et al., 2010; Cervantes-Ramírez et al., 2013). Therefore, these results indicate that excess Leu by itself does not affect $\mathrm{B}^{0} \mathrm{AT} 1$ expression, but the combined excesses of all BCAA appear to stimulate its expression in the jejunum and ileum.

System $\mathrm{B}^{0} \mathrm{AT} 1$ is similarly expressed in all segments (duodenum, jejunum, and ileum) of the small intestine of mouse, mainly at the tip of the villi (Romeo et al., 2006), but in humans $\mathrm{B}^{0} \mathrm{AT} 1$ expression increases from the duodenum to the ileum (Terada et al., 2005). No studies have examined the expression pattern of $\mathrm{B}^{0} \mathrm{AT} 1$ in different segments of the small intestine of pigs. In the present study, as observed in mice (Romeo et al., 2006), $\mathrm{B}^{0} \mathrm{AT} 1$ expression did not differ between the jejunum and ileum in pigs fed either the basal or the excess Leu diet. However, $\mathrm{B}^{0} \mathrm{AT} 1$ expression in pigs fed the excess LIV diet was $50 \%$ higher in the jejunum as compared to that in the ileum. This result suggests that dietary excess of all BCAA may change not only the expression of $\mathrm{B}^{0} \mathrm{AT} 1$ but also its site of expression.

System $b^{0}, A T$ is the major transporter for cationic AA in the apical membrane of the small intestine. It forms a hetero-dimer with the membrane protein rBAT (Palacin et al., 2001); 
however, $b^{0},{ }^{+} \mathrm{AT}$ is the catalytic unit (Reig et al., 2002). Transporter $\mathrm{b}^{0+}{ }^{+} \mathrm{AT}$ is mainly expressed in epithelial cells and functions as an antiporter exchanging Leu for Lys so that the absorption of Lys is coupled with the efflux of Leu (Pineda et a., 2004). As reported previously (Bröer et al., 2004; Hatzoglou et al., 2004), the expression of $\mathrm{b}^{0+}{ }^{+}$AT represents its functional activity. Thus, expression values observed in the present study are thought to reflect the abundance of its respective encoded protein. Previously, we reported that dietary excess of either Leu or Ile or both decreased $\mathrm{b}^{0},{ }^{+} \mathrm{AT}$ expression in the jejunum of growing pigs (García-Villalobos et al., 2012; Cervantes-Ramírez et al., 2013). In agreement, the expression of $b^{0}{ }^{+} A T$ in the jejunum of pigs fed the excess Leu diets in the present study was approximately $60 \%$ of that in pigs fed the basal diet. Furthermore, $b^{0}{ }^{+}$AT expression in pigs fed the excess LIV diet was only $22 \%$ of that in pigs fed the basal diet (Table 4). These results consistently show that not only excess Leu but also an excesses of all BCAA reduce the expression of $b^{0},{ }^{+} A T$ in the jejunum. In contrast, $\mathrm{b}^{0},{ }^{+} \mathrm{AT}$ expression in the ileum was not affected by excess Leu or LIV. According to Silk et al. (1985) most AA absorption takes place in the jejunum, and low amounts of free AA are expected to reach the ileum, suggesting a lowered requirement for AA transporters in the ileum. Indeed, the expression of $\mathrm{b}^{0}, \mathrm{AT}$ in the ileum was less than $10 \%$ as compared to that in the jejunum. Hence, we speculate that dietary differences in BCAA contents prevailed in the jejunum but disappeared in the ileum, which may explain the lack of effect of excess Leu or LIV on $\mathrm{b}^{0},{ }^{+} \mathrm{AT}$ expression in the ileum.

Table 4. Relative expression of cationic $\left(\mathrm{b}^{0},+\mathrm{AT}\right.$ and $\left.\mathrm{CAT} 1\right)$ and neutral $\left(\mathrm{B}^{0} \mathrm{AT} 1\right)$ amino acid transporters in small intestine, liver, and longissimus muscle of pigs fed a wheat-based diet with excess Leu or excess Leu, Ile, and Val (relative to expression values of pigs fed the basal diet).

\begin{tabular}{lccr}
\hline & & Dietary treatment $^{2}$ & \\
\cline { 2 - 4 } & Basal & Excess Leu & Excess LIV \\
\hline $\mathrm{b}^{0},+$ AT & & & 22 \\
Jejunum & 100 & 60 & 1 \\
Ileum & 100 & 309 & 205 \\
Liver & 100 & 278 & 163 \\
CAT1 & & 215 & 154 \\
Jejunum & 100 & 260 & 53 \\
Ileum & 100 & 24 & 187 \\
Liver & 100 & 280 & 268 \\
Longissimus & 100 & 147 & 150 \\
B $^{0}$ AT1 & 100 & 126 & \\
Jejunum & 100 & & \\
Ileum & 100 & & \\
\hline
\end{tabular}

abasal diet: wheat supplemented with crystalline amino acids; excess Leu: basal diet plus $0.43 \% \mathrm{~L}-\mathrm{Leu}$; excess LIV: basal diet plus $0.43 \%$ L-Leu, $0.20 \%$ L-Ile, and $0.25 \%$ L-Val.

Expression of CAT1 appears to be confined to the basolateral membrane (Cariappa et al., 2002), but mostly takes place in non-epithelial cells (Hatzoglou et al., 2004). We observed that CAT1 expression in the intestinal mucosa was markedly lower compared to $b^{0},{ }^{+} \mathrm{AT}$. CAT1 is strongly trans-stimulated by the concentration of cationic AA on the other side of the membrane and conducts transport in exchange for other cationic AA (Closs et al., 2004). The lower CAT1 expression in the jejunum observed in the present study may be explained by the adaptive regulation theory (Hatzoglou et al., 2004), which implies that sufficient AA supply inhibits while deficient supply stimulates CAT1 expression. The abundant $\mathrm{b}^{0},{ }^{+} \mathrm{AT}$ expression 
in jejunum may suggest high intra-enterocyte concentration (sufficient supply) of cationic AA, which in turn might inhibit CAT1 expression. In contrast, the low expression of $b^{0},{ }^{+} A T$ in the ileum, indicating a low intra-enterocyte concentration (deficient supply) of cationic AA, may have stimulated ileal CAT1 expression. Thus, the efflux of cationic AA from the enterocyte through CAT1 appears to be related to influx of these AA via $b^{0},{ }^{+}$AT. The lack of an effect of BCAA on CAT1 expression in the jejunum confirms the results of a previous study (Morales et al., 2013) and indicates that CAT1 is not directly related to the neutral AA.

Absorbed AA from dietary protein are transported to the liver via the portal vein where hepatocytes convert them into proteins, urea, and other metabolites, and export them to other tissues (Kilberg, 1982). Thus, transport of recently absorbed AA into hepatocytes is critical for adequate metabolism in the liver as well as in the entire animal. According to Rérat et al. (1992), liver cells do not retain BCAA, but large amounts of cationic AA (Lys and Arg) are taken up and retained by hepatocytes. There have been no studies of the effect of excess dietary BCAA on the expression of cationic AA transporters in hepatocytes. In the present study, $\mathrm{b}^{0}{ }^{+}$AT expression in the liver was not affected by excess Leu or excess LIV, but CAT1 expression levels in pigs fed the excess Leu or excess LIV diet were approximately 24 and $53 \%$, respectively, of that in pigs fed the basal diet. There is no clear explanation for the reduced expression of CAT1, although it may result from differences in the serum concentration of cationic AA, potentially caused by differences in dietary levels of BCAA.

Muscle growth in animals depends on the proper metabolism of absorbed AA, which is closely related to their transport into or out of muscle cells through specific transporters. According to Drummond et al. (2010), AA transporters play a key role in regulating muscle protein metabolism. Because Lys is the first limiting AA in practical diets for pigs and the most abundant AA in muscle proteins (Mahan and Shields Jr., 1998), its transport across the muscle cell membrane is of particular interest. Variations in CAT1 expression in the skeletal muscle of pigs due to different dietary AA levels have been reported. García-Villalobos et al. (2012) found that adequate, as compared to deficient levels of Lys, Thr, and Met, increased the expression of CAT1 in LD. In contrast, we found that excess Leu or excess LIV did not affect CAT1 expression in the LD. This may be an expected response because CAT1 system specifically transports cationic AA and does not interact with BCAA for transport of either group of AA.

The results of the present study, in which feed intake did not differ, reveal that excess Leu alone or combined with surplus Ile and Val in wheat-based diets differentially affects the expression of AA transporters in the studied tissues. Moreover, expression of AA transporters differed between the jejunum and ileum. Finally, changes observed in the intestinal expression of AA transporters in the present study are expected to affect the availability of AA, which in turn would affect the expression in other tissues, such as that detected in liver.

\section{ACKNOWLEDGMENTS}

The National Science and Technology Council of México (CONACYT) is acknowledged for providing scholarships to H. García and N. Arce. The authors thank UABC (Convocatoria Interna) and Evonik Industries for funding this project.

\section{REFERENCES}

Block KP (1989). Interactions among leucine, isoleucine, and valine with special reference to the branched-chain amino acid antagonism. In: Absorption and utilization of amino acids (Friedman M, ed.). CRC Press, Boca Raton, 229-244. 
Block KP and Harper AE (1984). Valine metabolism in vivo: effects of high dietary levels of leucine and isoleucine. Metabolism 33: 559-566.

Bröer A, Klingel K, Kowalczuk S, Rasko JE, et al. (2004). Molecular cloning of mouse amino acid transport system B0, a neutral amino acid transporter related to Hartnup disorder. J. Biol. Chem. 279: 24467-24476.

Bröer S (2008). Amino acid transport across mammalian intestinal and renal epithelia. Physiol. Rev. 88: 249-286.

Cariappa R, Heath-Monnig E, Furesz TC, Kamath SG, et al. (2002). Stable polarized expression of hCAT-1 in an epithelial cell line. J. Membr. Biol. 186: 23-30.

Cervantes-Ramírez M, Méndez-Trujillo V, Araiza-Piña BA, Barrera-Silva MA, et al. (2013). Supplemental leucine and isoleucine affect expression of cationic amino acid transporters and myosin, serum concentration of amino acids, and growth performance of pigs. Genet. Mol. Res. 12: 115-126.

Closs EI, Simon A, Vékony N and Rotmann A (2004). Plasma membrane transporters for arginine. J. Nutr. 134: 2752S-2767S.

Drummond M, Glynn EL, Fry CS, Timmerman KL, et al. (2010). An increase in essential amino acid availability upregulates amino acid transporter expression in human skeletal muscle. Am. J. Physiol. 298: E1011-E1018.

Edmonds MS and Baker DH (1987). Amino acid excesses for young pigs: effects of excess methionine, tryptophan, threonine or leucine. J. Anim. Sci. 64: 1664-1671.

García-Villalobos H, Morales-Trejo A, Araiza-Piña BA, Htoo JK, et al. (2012). Effects of dietary protein and amino acid levels on the expression of selected cationic amino acid transporters and serum amino acid concentration in growing pigs. Arch. Anim. Nutr. 66: 257-270.

Gatnau R, Zirnmerman DR, Nissen SL, Wannemuehlert M, et al. (1995). Effects of excess dietary leucine and leucine catabolites on growth and immune responses in weanling pigs. J. Anim. Sci. 73: 159-165.

Hatzoglou M, Fernandez J, Yaman I and Closs E (2004). Regulation of cationic amino acid transport: the story of the CAT-1 transporter. Annu. Rev. Nutr. 24: 377-399.

Kilberg MS (1982). Amino acid transport in isolated rat hepatocytes. J. Membr. Biol. 69: 1-12.

Langer S, Scislowski PWD, Brown DS, Dewey P, et al. (2000). Interactions among the branched-chain amino acids and their effects on methionine utilization in growing pigs: effects on plasma amino- and keto-acid concentrations and branched-chain keto-acid dehydrogenase activity. Br. J. Nutr. 83: 49-58.

Liao SF, Vanzant ES, Harmon DL, McLeod KR, et al. (2009). Ruminal and abomasal starch hydrolysate infusions selectively decrease the expression of cationic amino acid transporter mRNA by small intestinal epithelia of foragefed beef steer. J. Dairy Sci. 92: 1124-1135.

Mahan DC and Shields RG Jr (1998). Essential and nonessential amino acid composition of pigs from birth to 145 kilograms of body weight, and comparison to other studies. J. Anim. Sci. 76: 513-521.

Majumder M, Yaman I, Gaccioli F, Zeenko VV, et al. (2009). The hnRNAbinding proteins hnRNP L and PTB are required for efficient translation of the Cat-1 arginine/lysine transporter mRNA during amino acid starvation. Mol. Cell. Biol. 29: 2899-2912.

Méndez V, Avelar E, Morales A, Cervantes M, et al. (2011). A rapid protocol for purification of total RNA for tissues collected from pigs at a slaughterhouse. Genet. Mol. Res. 10: 3251-3255.

Morales A, Barrera MA, Araiza AB, Zijlstra RT, et al. (2013). Effect of excess levels of lysine and leucine in wheat-based, amino acid-fortified diets on the mRNA expression of two selected cationic amino acid transporters in pigs. J. Anim. Physiol. Anim. Nutr. 97: 263-270.

National Research Council, NRC (2012). Nutrient Requirements of Swine, 11th revised edn. National Academy Press, Washington, DC.

Norma Oficial Mexicana - NOM-062-ZOO-1999 (2001). Especificaciones técnicas para la producción, cuidado y uso de los animales de laboratorio (Ochoa MLI, eds.). Diario Oficial de la Federación, Ciudad de México.

Palacin M, Fernández E, Chillarón J and Zorzano A (2001). The amino acid transport system b $(0,+)$ and cystinuria. Mol. Membr. Biol. 18: 21-26.

Pineda M, Wagner CA, Bröer A, Stehberger PA, et al. (2004). Cystinuria-specific rBAT(R365W) mutation reveals two translocation pathways in the amino acid transporter rBATb0, AT. Biochem. J. 377: 665-674.

Reig N, Chillaron J, Bartoccioni P, Fernández E, et al. (2002). The light subunit of system $\mathrm{b}(0,+)$ is fully functional in the absence of the heavy subunit. EMBO J. 21: 4906-4914.

Rérat A, Simoes-Nuñes C, Mendy F, Vaissade P, et al. (1992). Splanchnic fluxes of amino acids after duodenal infusion of carbohydrate solutions containing free amino acids or oligopeptides in the non-anaesthetized pig. Br. J. Nutr. 68: 111-138.

Romeo E, Dave MH, Bacic D, Ristic Z, et al. (2006). Luminal kidney and intestine SLC6 amino acid transporters of B0AT-cluster and their tissue distribution in Mus musculus. Am. J. Physiol. 290: F376-F383.

Sambrook J and Russell DW (2001). Molecular Cloning: A Laboratory Manual. 3rd edn. Cold Spring Harbor Laboratory Press, Cold Spring Harbor. 
Silk DB, Grimble GK and Rees RG (1985). Protein digestion and amino acid and peptide absorption. Proc. Nutr. Soc. 44: 63-72.

Stein HH, Kim SW, Nielsen TT and Easter RA (2001). Standardized ileal protein and amino acid digestibility by growing pigs and sows. J. Anim. Sci. 79: 2113-2122.

Terada T, Shimada Y, Pan X, Kishimoto K, et al. (2005). Expression profiles of various transporters for oligopeptides, amino acids and organic ions along the human digestive tract. Biochem. Pharmacol. 70: 1756-1763.

Wiltafsky MK, Pfaff MW and Xaver F (2010). The effects of branched-chain amino acid interactions on growth performance, blood metabolites, enzyme kinetics and transcriptomics in weaned pigs. Br. J. Nutr. 103: 964-976.

Zhang S, Qiao S, Ren M, Zeng X, et al. (2013). Supplementation with branched-chain amino acids to a low-protein diet regulates intestinal expression of amino acid and peptide transporters in weanling pigs. Amino Acids 45: 1191-1205. 